\title{
Laparoscopy in penetrating abdominal stab wounds: Does it have a role?
}

\author{
Mohamed Riad, MD; Hatem Mohammed, MD; \\ Yasser Hussein, MD; Mohammad Lofty, MD \\ General Surgery Department Zagazig University
}

\begin{abstract}
Background: If the patient of penetrating abdominal stab wound is hemodynamically stable and has equivocal abdominal examination findings, surgeons pose a significant challenge to take decision in favor of surgery or nonoperative conservative treatment. This decision requires a precise diagnosis that is not always possible with imaging techniques. In consequence of this circumstance; laparoscopy can be a diagnostic or therapeutic tool in these cases. It also leads to avoid negative exploratory laparotomies.

Methods: This is a randomized study including 51 patients with penetrating abdominal stab wounds. The patients were selected according to the following criteria; hemodynamically stable, no signs of peritonitis and fully conscious without evidence of raised intracranial pressure and absence of contraindication for pneumoperitoneum. So we were able to evaluate the diagnostic and therapeutic role of laparoscopy.

Results: Patients were divided into two groups. Group I underwent exploratory laparotomy (26 patients). Group II underwent laparoscopic laparotomy (25 patients). The mean operating time in both groups was $(89 \pm 23.5$ vs. $55 \pm 19$ minutes, $(P<0.05)$ respectively. The length of hospital stay was (9.3 vs. 4.1 days, $P<0.05)$ respectively in both groups. Postoperative morbidity in both groups was $(19.23 \%$ vs. $12 \%, P>0.05)$ respectively. There was no mortality in both groups. Accurate diagnosis was done to all patients in Group II except one patient (96.15\%) with missed intestinal injury (this happened in the first cases of the study). Treatment was done for four patients from eight patients with discovered organ injuries (50\%). Other four patients were converted to exploratory laparotomy (50\%) due to severe injuries.

Conclusion: The use of laparoscopy as a diagnostic or therapeutic method in patients with penetrating abdominal stab wounds is an efficient, safe and effective method. The most important advantages are reduction of morbidity, shortening of hospitalization and cost-effectiveness.

Key words: Penetrating abdominal stab trauma, laparoscopy, laparotomy.
\end{abstract}

\section{Introduction:}

Penetrating injuries of the abdomen are traditionally treated with exploratory laparotomy. The high non-therapeutic laparotomy rate and associated morbidity after mandatory laparotomy for abdominal stab wounds led to the current selective non-operative management strategy. ${ }^{1-3}$ It is reported that $50-70 \%$ of abdominal stab injuries penetrate the peritoneum, but
$20-40 \%$ of these penetrating wounds do not lead to significant organ injury. ${ }^{4}$ Despite the growing use of diagnostic methods such as abdominal ultrasonography (US), computed tomography (CT), diagnostic peritoneal lavage (DPL), intravenous pyelography (IVP), and angiography, it is still difficult to evaluate the severity of penetrating abdominal stab wounds. ${ }^{5}$

Patients with penetrating abdominal stab 
wounds are managed non-operatively in many centers, despite the fact that an explorative laparotomy may be an appropriate treatment strategy in selected patients. The criteria defining when an explorative laparotomy is appropriate state that hemodynamic instability and signs of peritonitis must be present. Hemodynamically stable patients without signs of peritonitis may be carefully followed non-operatively. 6,7

Although good results can be obtained with observation, there are inherent limitations to this approach. Observation requires that serial abdominal examinations be performed by an experienced surgeon able to detect subtle changes in physical findings (who may not be available at every time). Observation also requires hospital admission for several days, taxing the resources of overburdened institutions. ${ }^{8}$ These reasons have prompted the search for a less invasive and more reliable method of evaluation. This is the basis of laparoscopy for abdominal trauma. ${ }^{9}$

\section{Patients and methods:}

This study included 51 patients who were admitted to the emergency unit of Zagazig University hospitals during a period from April 2010 to October 2012. The patients were divided randomly into two groups: group I included patients who underwent exploratory laparotomy and group II included patients who underwent laparoscopic exploration.

Preoperatively all patients were submitted to full history, careful general and abdominal examination, abdominal ultrasonography, laboratory tests and chest $\mathrm{x}$-ray (for patients with stab wounds in the upper abdomen to exclude peumothorax).

The following criteria were followed for patients' selection: penetrating stab injuries in the abdomen with stable vital signs, no signs of peritonitis and ability to give informed consent for the study added to them intact sensations without evidence of raised intracranial pressure and absence of contraindication for pneumoperitoneum (i.e. cardiopulmonary disease).

\section{Surgical Procedure:}

In preparing the patient for laparoscopic exploration, the usual rules of trauma care were followed, including adequate intravenous access, Foley catheterization, and stomach decompression with N/G tube. The procedure was conducted under general anesthesia and patients were prepped in the supine position from chin to thighs for a possible conversion to an open procedure. The operating table allowed Trendelenburg, reverse Trendelenburg positions, and side-toside tilting of the table.

The entrance site of the stab was sutured and covered with an occlusive dressing to allow for creation of pneumoperitoneum. The first trocar for $10 \mathrm{~mm}$ laparoscope $\left(30^{\circ}\right)$ was inserted at the umbilicus. After the abdomen has been entered, the anterior abdominal wall at the site of the stab (that was depressed with a finger) was examined to detect peritoneal violation. If the peritoneum was intact the procedure was terminated. If there was violation of the peritoneum another two $5 \mathrm{~mm}$ trocars were inserted at the right and left paramedian sites at the level of the umbilicus for systemic examination of the abdomen using atraumatic bowel graspers. If no injury was identified, the procedure was terminated and the patient was put under observation. If injury was found, it was either managed laparoscopically or with exploratory laparotomy.

Figure(1) shows the used algorithm for the laparoscopic management of abdominal stab wounds [adapted from Choi and Lim]. ${ }^{10}$ 


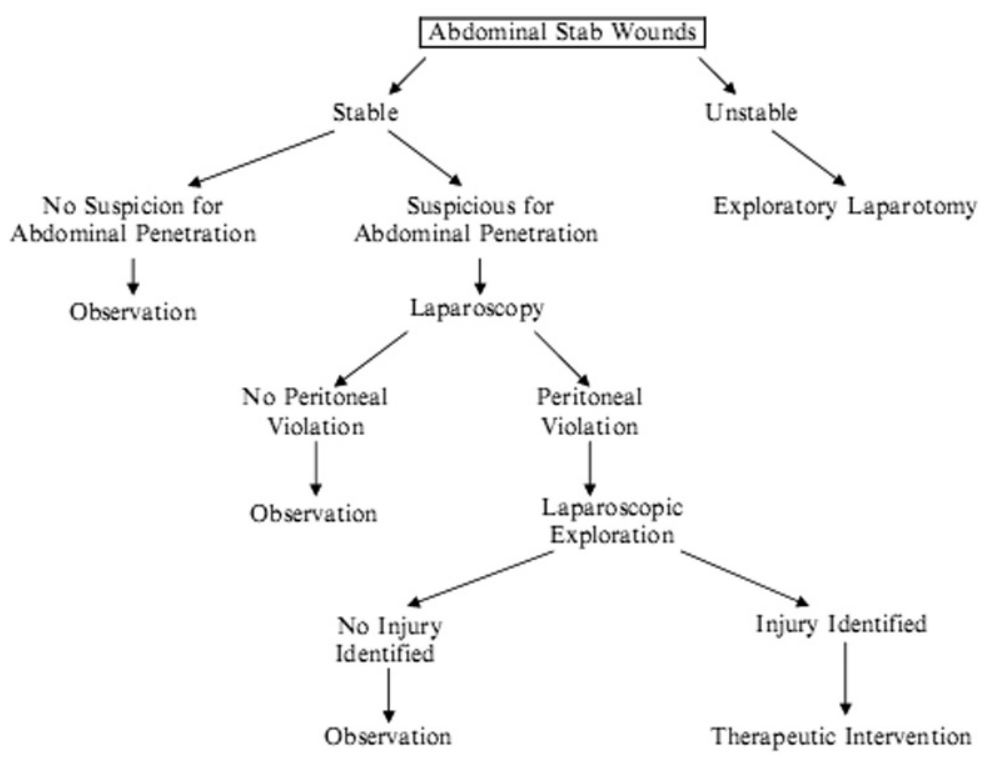

Figure (1): Algorithm for the laparoscopic management of abdominal stab wounds.

\section{Results:}

This study was conducted on 51 patients with penetrating abdominal stab wounds. They were divided into two groups and underwent operations either exploratory laparotomy for group I or laparoscopic exploration for group II. Group I included 26 patients (about 51\%) and group II included 25 patients (about $49 \%$ ). The mean age in group I was $25 \pm 3.5$ years; 23 patients were males $(88.5 \%)$ and 3 females $(11.5 \%)$. The mean age in group II was $28 \pm 2.3$ years; all patients were males in this group.

The patients were divided into three categories according to results of exploration. $1^{\text {st }}$ patients having no peritoneal penetration, $2^{\text {nd }}$ patients having peritoneal penetration without intra-abdominal organ injuries, and $3^{\text {rd }}$ patients having peritoneal penetration with intra-abdominal organ injuries, as shown in Table(1).

Table (1): Results of exploration in both groups.

\begin{tabular}{|l|c|c|}
\hline Results & Group I & Group II \\
\hline No. of patients & $26(23$ male) & $25($ all male $)$ \\
\hline No peritoneal penetration & $8(30.77 \%)$ & $7(28 \%)$ \\
\hline Peritoneal penetration without intra-abdominal organ injuries & $11(42.3 \%)$ & $10(40 \%)$ \\
\hline Peritoneal penetration with intra-abdominal organ injuries & $7(26.92 \%)$ & $8(32 \%)$ \\
\hline
\end{tabular}

In group II; all patients were accurately diagnosed except in one case with missed intestinal injury (96.15\%); this happened in the first cases of the study. This perforation was found in the posterior gastric wall, which was managed by exploratory laparotomy which done 2 days later; when signs of peritonitis developed.

In group I, there were seven patients having peritoneal penetration with intra-abdominal organ injuries $(26.92 \%)$; three patients had small intestinal injury only, two patients had small intestinal injury with mesenteric injury and two patients had liver injury. On 
the other hand, in group II there were eight patients having peritoneal penetration with intra-abdominal organs injuries (32\%); three patients having small intestinal injury only, two patients having small intestinal injury with mesenteric injury, one patient had colonic injury, one patient had liver injury and one patient has splenic injury, as shown in Table(2).

Table (2): Shows the type of injury in both groups on initial evaluation.

\begin{tabular}{|l|c|c|}
\hline Type of injury & Group I & Group II \\
\hline Small intestinal injury & 3 & 3 \\
\hline Small intestinal and mesenteric injury & 2 & 2 \\
\hline Colonic injury & 0 & 1 \\
\hline Liver injury & 2 & 1 \\
Splenic injury & 0 & 1 \\
\hline
\end{tabular}

Four cases (50\%) in group II were managed laparoscopically: Two cases with single small intestinal injury (less than $1 \mathrm{~cm}$ ) were managed with intra-corporeal suturing. In one case with small intestinal and mesenteric injury; the intestinal injury was managed with intra-corporeal suturing due to the small wound size and the mesenteric injury was also small and was controlled with clips used for laparoscopic cholecystectomy (additional $10 \mathrm{~mm}$ port was added in the supra-pubic region). The $4^{\text {th }}$ case had a liver tear that was superficial and was controlled with bipolar diathermy coagulation and compression by a piece of gauze for ten minutes.

The other four cases who were converted to open laparotomy: $1^{\text {st }}$ case had splenic injury which was near to the hilum with blood clot over it and open splenectomy was done. $2^{\text {nd }}$ case had intestinal injury with big mesenteric injury with sign of ischemia at the affected intestinal part; resection-anastomosis was done. $3^{\text {rd }}$ case had colonic injury (transverse colon) with faecal soiling, in this patient colostomy was done at the perforation site. $4^{\text {th }}$ case, had multiple small bowel perforations that needed resectionanastomosis of the affected segment.

Postoperative morbidity in group I was recorded in five patients, while in group II it was found in three patients (19.23\% vs. $12 \%, \mathrm{P}>0.05)$. There was a significant difference between both groups regarding mean operative time; in group I it was $89 \pm 23.5$ minutes, while in group II it was $55 \pm 19(\mathrm{P}<0.05)$. The length of hospital stay was (9.3 vs. 4.1 days, $\mathrm{P}<0.05)$ respectively in both groups. In consequence, there was decreasing postoperative morbidity and length of hospital stay in group II that led to improved cost-effectiveness. No mortality was detected in both groups. Postoperative characters of patients are showed in Table(3). 
Table (3): Postoperative characters.

\begin{tabular}{|l|c|c|c|}
\hline Postoperative characters & Group I & Group II & P value \\
\hline Mean operative time (minutes) & $89 \pm 23.5$ & $55 \pm 19$ & $<0.05$ \\
\hline Length of stay in the hospital (days) & 9.3 & 4.1 & $<0.05$ \\
\hline Postoperative morbidity & $5(19.23 \%)$ & $3(12 \%)$ & $>0.05$ \\
1-Seroma & 1 & 1 & \\
2-Wound infection & 1 & 0 & \\
4-Ileus & 2 & 1 & \\
5-Chest infection & 1 & 1 & \\
\hline
\end{tabular}

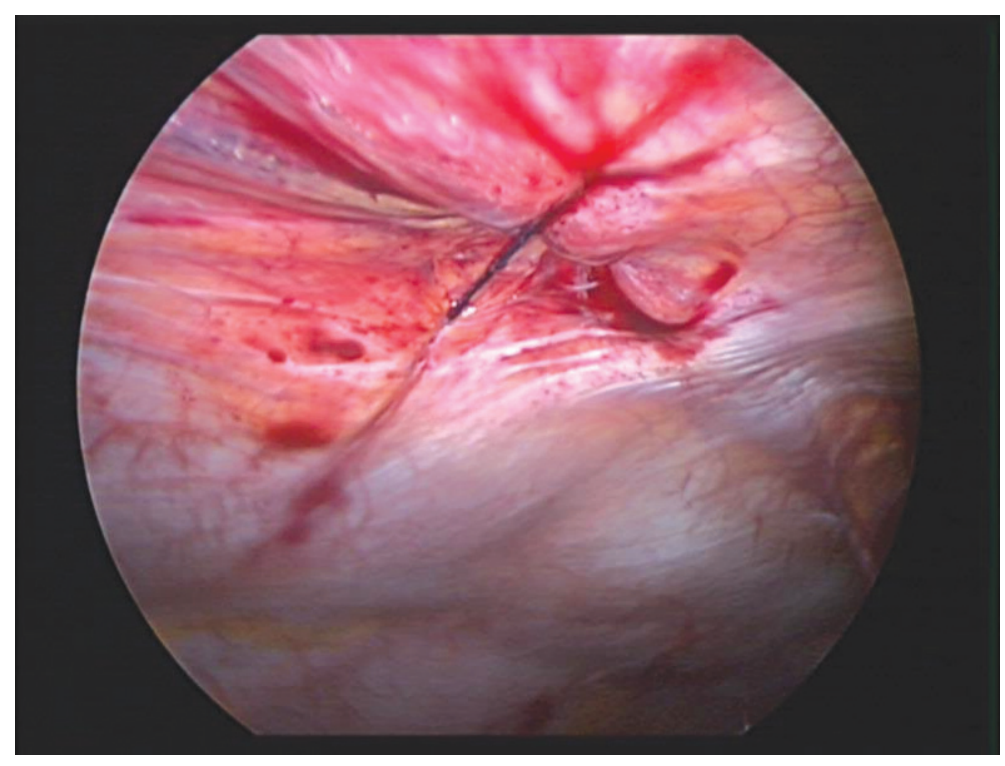

Figure (2): Peritoneal stab perforation.

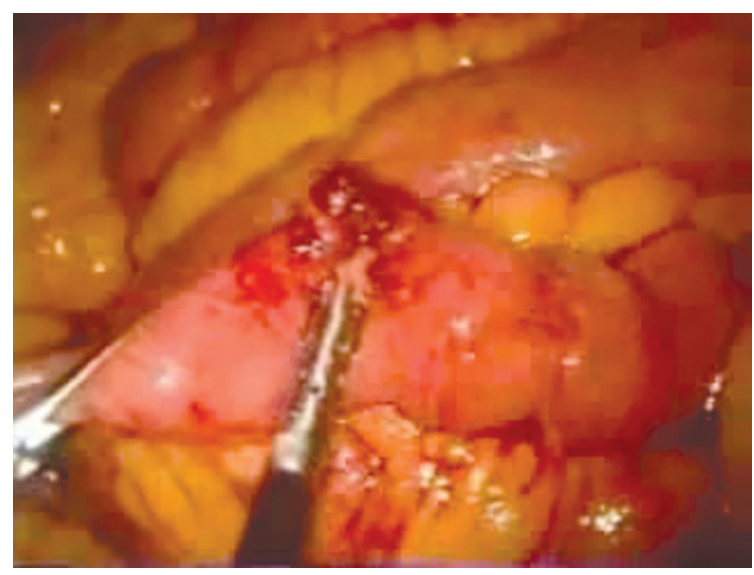

Figure (3): Small intestinal stab perforation.

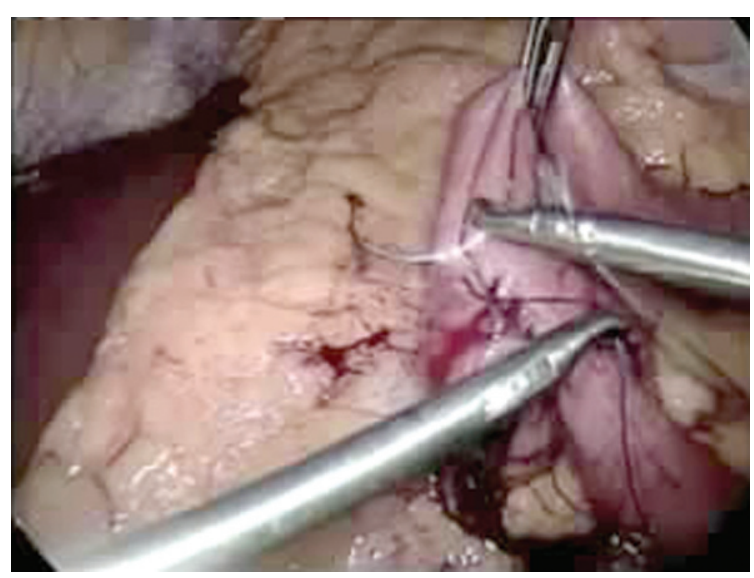

Figure (4): Intra-corporeal intestinal repair. 


\section{Discussion:}

Laparoscopy raises many safety concerns in the trauma setting, including tension pneumothorax while the patient is under anesthesia, increase in intracranial pressure, and gas embolism induced by pneumoperitoneum, not to mention the risk of missed injuries and medico-legal consequences. ${ }^{11-13}$ Although these risks are real, they can be reduced by careful selection of patients, judicious use of chest tubes, and meticulous technique. Although stable vital signs do not preclude major internal injuries requiring prompt repair, initial laparoscopy would yield further localizing data and should not significantly delay open operative treatment. 14

In the current study, included 51 patients; 15 of those patients did not have peritoneal penetration (29.41\%) and 20 patients (39.21\%) had peritoneal penetration without intraabdominal injury (the missed case of gastric perforation from group II was excluded) this coincides with what is stated by Thal and Fabian et $\mathrm{al}^{4,15}$ who said; approximately one third of patients presenting with anterior abdominal wall stab wounds actually sustain an injury that requires surgical intervention. This finding is related to the fact that $25 \%$ of anterior abdominal wall stab wounds do not penetrate the peritoneal cavity, but approximately one half of wounds that do violate the peritoneum cause visceral injury requiring surgical repair.

In our study, we found; non-therapeutic laparotomy in group I was about $73.1 \%$ (19 patients from 26 patients) this is more than the ranges reported by other reviews that the incidence of unnecessary laparotomy rates range from $23 \%$ to $65 \%$ in patients presenting with abdominal wall stab wounds. $4,9,16-19$

On the other hand, non-therapeutic exploratory laparotomy could be avoided in 16 patients from group II $(64 \%)$, this is near to the results of Ahmed et al and others ${ }^{14,20,21}$ who suggested that, when laparoscopy is deployed in the algorithm for management of penetrating injuries of the abdomen, nontherapeutic exploratory laparotomy can be avoided in $55-87 \%$ of trauma patients.
The laparoscopic success rate to diagnose peritoneal penetration with or without intraabdominal injury was $(96.15 \%)$.

There was one case of missed intestinal injury $(4 \%)$ this a little more than what is reported by Kopelman et al ${ }^{19}$ in their study (on 38 patients) that missed injuries incidence was $0 \%$ and much less than what is reported by Fabian et al and Rossi et al ${ }^{4,22}$ who reported missed injury rate of $40 \%$. This missed case in our study could be referred to our starting experience in that new approach.

In group II, 4 cases (50\%) hwo needed surgical intervention; could be managed laparoscopically. This percentage is much less than what was reported by Lin et $\mathrm{al}^{9}$ who stated a success rate of laparoscopic repair $94.1 \%$ and referred their high success rate to that their attending surgeons were highly skilled in advanced laparoscopic techniques.

By adding these 4 cases treated laparoscopically to the other cases that did not need intervention, the total number of patients that avoided exploratory laparotomy in group II became 20 patients (out of 25 patients) $(80 \%)$. Although most of our patients did not have long term follow-up we agree with Ahmed et $\mathrm{al}^{14}$ that there will be reduction in the incidence of hernias, adhesions and intestinal obstruction.

There was no significant difference in the incidence of postoperative complications in bothgroupsincludedinthestudy, and thisagreed with Leppaniemi A. and Haapiainen R. 21 There was a significant difference as regard the average hospital stay between the two groups $(\mathrm{P}<0.05)$ in favor of group II (the average hospital stay in group I was 9.3 days and in group II it was 4.1 days).

The shorter length of hospital stay in group I than in group II reflects the nature of minimal invasiveness of the laparoscopic approach. With respect to outcomes, most authors reported a decreased complication rate, shorter length of hospital stay, and decreased costs when negative laparoscopy is compared with a negative or non-therapeutic open laparotomy. 17,20,23-26

We agree with Lin et al ${ }^{9}$ that visualization of solid organs is simple to perform and is 
reliable, but complete examination of the intestines presents a greater challenge for surgeons. The key points to avoid missed injuries in diagnostic laparoscopy include the following: (1) a systematic and careful inspection; (2) changing the patient's position during laparoscopic procedures for inspection; and (3) use of atraumatic grasping forceps for bowel manipulation. And the keys to success in therapeutic laparoscopy include the following: (1) appropriate change of the patient's posture; (2) careful planning of port placement; and (3) technical ability in advanced laparoscopic procedures.

\section{Conclusion:}

From the data collected in this study we can conclude that diagnostic laparoscopy is feasible, effective and safe procedure for hemodynamically stable patients with abdominal stab wounds. Not only because of shorter hospital stay but also the complications from non-therapeutic laparotomies and delayed treatment of significant intra-abdominal injuries may be minimized. Therapeutic laparoscopy also can be applied for selected patients but it demands from the trauma surgeons to have advanced laparoscopic skills.

\section{References:}

1- Demetriades D, Rabinowitz B: Indications for operation in abdominal stab wounds; a prospective study of 651 patients. Ann Surg 1987; 205: 129-132.

2- Sirinek K, Page C, Root $\mathrm{H}$, et al: Is exploratory celiotomy necessary for all patients with truncal stab wounds? Arch Surg 1990; 125: 844-848.

3- Leppaniemi A, Salo J, Haapiainen R: Complications of negative laparotomy for truncal stab wounds. J Trauma 1995; 38: 54-58.

4- Fabian T, Croce M, Stewart R, et al: A prospective analysis of diagnostic laparoscopy in trauma. Ann Surg 1993; 217 (5): 557-564.

5- Alimoglu M, Yucel L, Sabasi E, et al: Evolution-based algorithm for the management of penetrating abdominal stab injury. Eur J Trauma Emerg Surg 2012; 12:188-8.

6- Como J, Bokhari F, Chiu W, et al: Practice management guidelines for selective nonoperative management of penetrating abdominal trauma. J Trauma 2010; 68: 721-733.

7- Demetriades D, Hadjizacharia P, Constantinou C, et al: Selective nonoperative management of penetrating abdominal solid organ injuries. Ann Surg 2006; 244: 620-628.

8- Weinberg J, Magnotti L, Edwards N, et al: "Awake" laparoscopy for the evaluation of equivocal penetrating abdominal wounds. Injury, Int J Care Injured 2007; 38: 60-64.

9- Lin $\mathrm{H}, \mathrm{Wu} \mathrm{J}, \mathrm{Tu} \mathrm{Ch}$, et al: Value of diagnostic and therapeutic laparoscopy for abdominal stab wounds. World J Surg 2010; 34: 1653-1662.

10-Choi Y, Lim K: Therapeutic laparoscopy for abdominal trauma. Surg Endosc 2003; 17: 421-427.

11-Elliott D, Rodrigues A, Moncure M, et al: The accuracy of diagnostic laparoscopy in trauma patients; a prospective controlled study. Intern Surg 1998; 83: 294-298.

12-Murray J, Demetriades D, Arsenio J, et al: Occult injuries to the diaphragm: Prospective evaluation of laparoscopy in penetrating injuries to the left lower chest. J Am Coll Surg 1998; 187: 626-630.

13-Villaricencio R, Aucar J: Analysis of laparoscopy in trauma. $J$ Am Coll Surg 1999; 189: 11-20.

14-Ahmed N, Whelan J, Brownlee J, et al: The contribution of laparoscopy in evaluation of penetrating abdominal wounds. $J \mathrm{Am}$ Coll Surg 2005; 201: 213-216.

15-Thal E: Evaluation of peritoneal lavage and local exploration in lower chest and abdominal stab wounds. J Trauma 1977; 17: 642-648

16-Ditmars M, Bongard F: Laparoscopy for triage of penetrating trauma: The decision to explore. J Laparoendosc Surg 1996; 6: 285-291.

17-Hallfeldt K, Trupka A, Erhard J, et al: Emergency laparoscopy for abdominal 
stab wounds. Surg Endosc 1998; 12: 907-910.

18-Ertekin C, Yanar H, Taviloglu K, et al: Unnecessary laparotomy by using physical examination and different diagnostic modalities for penetrating abdominal stab wounds. Emerg Med J 2005; 22: 790-794.

19-Kopelman T, O’Neill P, Macias L, et al: The utility of diagnostic laparoscopy in the evaluation of anterior abdominal stab wounds. The American Journal of Surgery 2008; 196: 871-877.

20-DeMaria E, Dalton J, Gore D, et al: Complementary roles of laparoscopic abdominal exploration and diagnostic peritoneal lavage for evaluating abdominal stab wounds: A prospective study. J Laparoendosc Adv Surg Tech A 2000; 10: 131-136.

21-Leppaniemi A, Haapiainen R: Diagnostic laparoscopy in abdominal stab wounds: A prospective, randomized study. $J$ Trauma 2003; 55: 636-645.

22-Rossi P, Mullins D, Thal E: Role of laparoscopy in the evaluation of abdominal trauma. Am J Surg 1993; 166 : 707-711.

23-Zantut L, Ivatury R, Smith R, et al: Diagnostic and therapeutic laparoscopy for penetrating abdominal trauma: A multi-center experience. J Trauma 1997; 42: 825-831.

24-Taner A, Topgul K, Kucukel F, et al: Diagnostic laparoscopy decreases the rate of unnecessary laparotomies and reduces hospital costs in trauma patients. $J$ Laparoendosc Adv Surg Tech A 2001; 11: 207-211.

25-Marks J, Youngelman D, Berk T: Cost analysis of diagnostic laparoscopy vs laparotomy in the evaluation of penetrating abdominal trauma. Surg Endosc 1997; 11: 272-276.

26-Sosa J, Baker M, Puente I, et al: Negative laparotomy in abdominal gunshot wounds: potential impact of laparoscopy. J Trauma 1995; 38: 194-197. 